\title{
Anti-CD32B Monoclonal Antibody BI-1206
}

National Cancer Institute

\section{Source}

National Cancer Institute. Anti-CD32B Monoclonal Antibody BI-1206. NCI Thesaurus. Code C156458.

A fully human monoclonal antibody targeting the Fc gamma receptor IIB (FcgRIIB; CD32B) with potential immunomodulatory and antineoplastic activities. Upon intravenous administration, anti-CD32B monoclonal antibody $\mathrm{BI}-1206$ selectively binds to CD32B, a receptor expressed on the surface of B-cells. This prevents CD32B-mediated internalization of anti-CD20 monoclonal antibodies, such as rituximab, which abrogates tumor cell resistance caused by CD32B-mediated monoclonal antibody internalization and degradation of CD32B-expressing B-cells. By blocking CD32B, BI-1206 may recover and enhance the activity of rituximab and other anti CD20 monoclonal antibodies. In addition, $\mathrm{BI}-1206$ itself activates the immune system to exert an immune-mediated tumor cell death of B-cells. CD32B, an inhibitory member of the FcgammaR family, is implicated in immune cell desensitization and tumor cell resistance. 